\title{
Soziale Ungleichheit und Sozialpolitik
}

\author{
Claudia Zilla
}

Lateinamerika ist im weltweiten Vergleich die Region mit der stärksten Ausprägung sozialer Ungleichheit. Die durchschnittliche Armutsrate in der Region ist zwar nicht die höchste der Welt, jedoch viel zu hoch angesichts des erreichten Entwicklungsniveaus und des Einkommens pro Kopf. Im Verlauf des vergangenen Jahrzehnts wurde allerdings der Trend zunehmender sozialer Exklusion der 1990er Jahre umgekehrt. Armut und soziale Ungleichheit konnten dank hoher Wachstumsraten und der Steigerung der Sozialausgaben signifikant verringert werden. Ähnliche Entwicklungen sind auch in Brasilien zu beobachten. In diesem Sinne stellt das Land keine Ausnahme im regionalen Kontext dar. Allerdings lässt die Kombination von vier Merkmalen Brasilien zu einem besonders erfolgreichen Fall werden. Erstens gehörte Brasilien traditionell zur Gruppe der Staaten mit der größten sozialen Ungleichheit in der Region, so dass die Ausgangslage besonders kritisch war. Zweitens verzeichnete Brasilien zwischen 2003 und 2013 nur moderate Wirtschaftswachstumsraten, die unterhalb des lateinamerikanischen Durschnitts lagen. So war ein wichtiger fördernder Faktor in Brasilien nicht so günstig ausgeprägt wie in anderen Volkswirtschaften auf dem Subkontinent. Dennoch konnte Brasilien drittens in diesem Zeitraum herausragende Erfolge bei der Reduzierung der Armut und der sozialen Ungleichheit erzielen, welche die Leistungen der übrigen Länder übertrafen. Viertens schließlich setzte diese positive soziale Entwicklung in Brasilien bereits Ende der 1990er Jahre und damit früher als in den meisten lateinamerikanischen Staaten ein.

"Wachstum mit Inklusion" heißt die Formel, die ein Novum in der sozioökonomischen Geschichte Brasiliens darstellt und für welche die Regierungen von Luiz Inácio Lula da Silva (2003-2010) und Dilma Rousseff (seit 2011), beide von der Arbeiterpartei (Partido dos Trabalhadores, PT), nicht nur im Inland, sondern auch in Lateinamerika und weltweit große Anerkennung erhalten. Brasilien wurde von einem Entwicklungsland zu einer "Entwicklungsmacht", die ihre entwicklungspolitische Expertise im Rahmen der internationalen Zusammenarbeit als emerging donor sogar auBerhalb der nationalen Grenzen verwertet (Zilla/Harig 2012). Brasilien gilt heute als eine Demokratie - die drittgrößte der Welt -, in der mit einer 
zielgerichteten Sozialpolitik die sozioökonomische Teilhabe der Bevölkerung stark ausgebaut werden konnte.

Die folgenden Ausführungen befassen sich mit den brasilianischen sozialen Errungenschaften des letzten Jahrzehnts, mit den relevantesten der dafür verantwortlichen Faktoren sowie mit den Defiziten und Herausforderungen, die auf dem Weg zu einer Industriegesellschaft für das südamerikanische Land weiterhin bestehen. Dabei wird jeweils einleitend der lateinamerikanische Kontext dargestellt, zu dem Brasilien gehört, aus dem das Land aber auch herausragt. Ausgeklammert werden die nationalen und internationalen Aspekte, die das Wirtschaftswachstum ermöglichten, sowie die parteipolitischen Kräfteverhältnisse und Entscheidungen, die der umgesetzten Sozialpolitik zugrunde liegen. Diese Dimensionen werden in anderen Beiträgen dieses Bandes behandelt.

\section{Soziale Errungenschaften \\ Lateinamerikas Erfolge}

Im Zentrum des Entwicklungsdiskurses der 1990er Jahre in Lateinamerika standen zwei Überzeugungen. Zum einen ging es um den Glauben an die Notwendigkeit der Sequenz "zunächst Wirtschaftswachstum, dann Umverteilung". Damit verbunden war zum anderen die Erwartung oder Hoffnung, dass Umverteilung überwiegend durch den trickle-downeffect, also das (natürliche) Durchsickern von Wohlstand in die unteren Schichten der Gesellschaft erfolgen würde oder sollte (Zilla 2012: 46f.). Ein 'minimalistisches' Rollenverständnis des Staates setzte sich durch: Er solle zwar (zumeist durch eine angebotsorientierte Wirtschaftspolitik) die für die ökonomische Entwicklung notwendigen Rahmenbedingungen schaffen, sich aber wenig regulierend bzw. sozialpolitisch 'sparsam' verhalten. Im Kontext des Washington Consensus, also des Maßnahmenbündels, das unter anderem Wechselkurskorrekturen, Fiskaldisziplin, Kürzungen der Sozialausgaben, eine Liberalisierung der Handelspolitik, die Deregulierung der Märkte, die Privatisierung von Staatsunternehmen und den Abbau von Subventionen umfasste, wurde der Staat verkleinert und zog sich aus ökonomischen und sozialen Bereichen zurück. Allerdings ging das lateinamerikanische Wirtschaftswachstum der 1990er Jahre in den meisten Ländern mit einer Steigerung der sozialen Ungleichheit einher. 
Diese kritische Korrelation änderte sich erst ab 1998 und wandelte sich ab 2002 definitiv zu einer günstigen; Wachstum fiel fortan mit einer Verringerung der sozialen Ungleichheit zusammen (Gasparini/Cruces 2013: 54). Durch die analytische Isolierung der Faktoren Wirtschaftswachstum und Umverteilung kann ihre jeweilige Auswirkung auf die Armutsbekämpfung ermittelt werden. Während in den 1990er Jahren das Wirtschaftswachstum fünf Prozent der Reduzierung und die (regressive) Umverteilung zwei Prozent der Steigerung der Armut erklärten, führten in den 2000er Jahren sowohl das Wirtschaftswachstum (sechs Prozent), als auch die (progressive) Umverteilung (vier Prozent) zu einem Rückgang der Armutsrate (Gasparini/Cruces 2013: 55).

\section{Brasiliens Leistungen}

Im lateinamerikanischen Kontext avancierte Brasilien in der letzten Dekade zum Musterbeispiel für die Förderung einer sozial inklusiven und mobilen Gesellschaft. Obwohl diese Leistung zumeist den PT-Regierungen gänzlich zugeschrieben wird, setzte die Verbesserung der sozioökonomischen Indikatoren bereits nach der Einführung des Plano Real im Jahr 1994 ein, unter der Regierung von Fernando Henrique Cardoso (1995-2002) von der Partei der Brasilianischen Sozialdemokratie (Partido da Social Democracia Brasileira, PSDB). Spürbare Resultate der Währungsreform und der begleitenden wirtschaftspolitischen Maßnahmen, die nach dem Machtwechsel 2003 von den darauffolgenden Regierungen beibehalten wurden, waren unter anderem die Bekämpfung der Hyperinflation und die Stabilisierung der Währung. Auch viele der positiven Entwicklungen der 2000er Jahre, wie der Anstieg der Exporte und somit des Handelsbilanzüberschusses, der ausländischen Direktinvestitionen, der Währungsreserven, der inländischen Bankkredite sowie niedrige Zinsen und geringe Arbeitslosigkeit, sind zum Teil als Folgen von Entscheidungen zu sehen, die zu früheren Zeitpunkten getroffen wurden. Zusammen mit einer aktiveren Sozialpolitik führten diese zu einer erheblichen sozioökonomischen Verbesserung im Lande (Trebat 2013: 130). So konnte zwischen der Einführung des Real 1994 und 2010 die Armutsrate um 67 Prozent verringert werden (Neri 2011: 27). Aber auch die Diskrepanz zwischen Armen und Reichen ist in Brasilien kleiner geworden. 


\section{Armut}

Rund 197 Millionen Menschen leben in Brasilien. Mit einem Bruttoinlandseinkommen (BIP) pro Kopf von 10.720 US-Dollar ist es ein Land oberen mittleren Einkommens (Weltbank 2011). Nach Daten der Comisión Económica para América Latina (CEPALSTAT 2013) ging der Anteil der in Armut lebenden Menschen von 38,7 Prozent im Jahr 2003 auf 24,9 Prozent im Jahr 2009 zurück. Bei der extremen Armut sank der Wert von 13,9 auf 7,0 Prozent.

Auch die Arbeitslosigkeit konnte zwischen 2003 und 2012 signifikant reduziert werden, von 12,3 auf 5,5 Prozent (CEPALSTAT 2013). Der Anstieg der Beschäftigung ging mit einer zunehmenden Formalisierung des Arbeitsmarktes einher. Dies bedeutet eine Qualitätssteigerung der Arbeit bzw. der Arbeitsplätze, die nun vom Arbeitsrecht und der sozialen Sicherung erfasst werden. Zur Armutsbekämpfung trug aber nicht nur das Wirtschaftswachstum bzw. die niedrigere Arbeitslosigkeit, sondern auch die Verringerung der Ungleichheit bei.

\section{Ungleichheit}

Brasilien verfügt traditionell über einen wenig ehrenhaften Ruf als eine sehr ungerechte Gesellschaft. Der brasilianische Gini-Koeffizient, also jener Index, der die Gleichverteilung $(=0)$ bzw. die Ungleichverteilung $(=1)$ des Einkommens misst, hat bisweilen weltweite Rekordwerte erreicht (Lustig/ López-Calva/Ortiz-Juárez 2012: 6). Nachdem dieser in den 1970er und 1980er Jahren zunahm und sich in den 1990er Jahren kaum veränderte, begann er ab 1998 und noch stärker ab 2002 zurückzugehen. Im Zeitraum 2001-2011 konnte der Gini-Koeffizient von 0,639 auf 0,559 reduziert werden. Dies stellt eine erhebliche Verringerung dar, die in der Welt ihresgleichen sucht. Damit hat Brasilien aufgehört, "Champion der Ungleichheit" in Lateinamerika zu sein. Der Gini-Koeffizient Brasiliens ist heute jedoch immer noch weit entfernt vom Durchschnitt der Länder der Organisation for Economic Co-operation and Development (OECD), der bei 0,31 liegt.

Die Einkommensschere schließt sich, wenn das Einkommen der ärmeren Zehntel der Gesellschaft schneller ansteigt als das der reicheren. Das bedeutet Wachstum zugunsten der Armen (crescimento pró-pobre), eine Entwicklung, die dem lateinamerikanischen Trend der 1990er Jahre entgegensteht und im Brasilien der 2000er Jahre stark ausgeprägt war. 
Grafik 1: Veränderung des Pro-Kopf-Einkommens zwischen 2001 und 2009 nach Einkommensgruppen

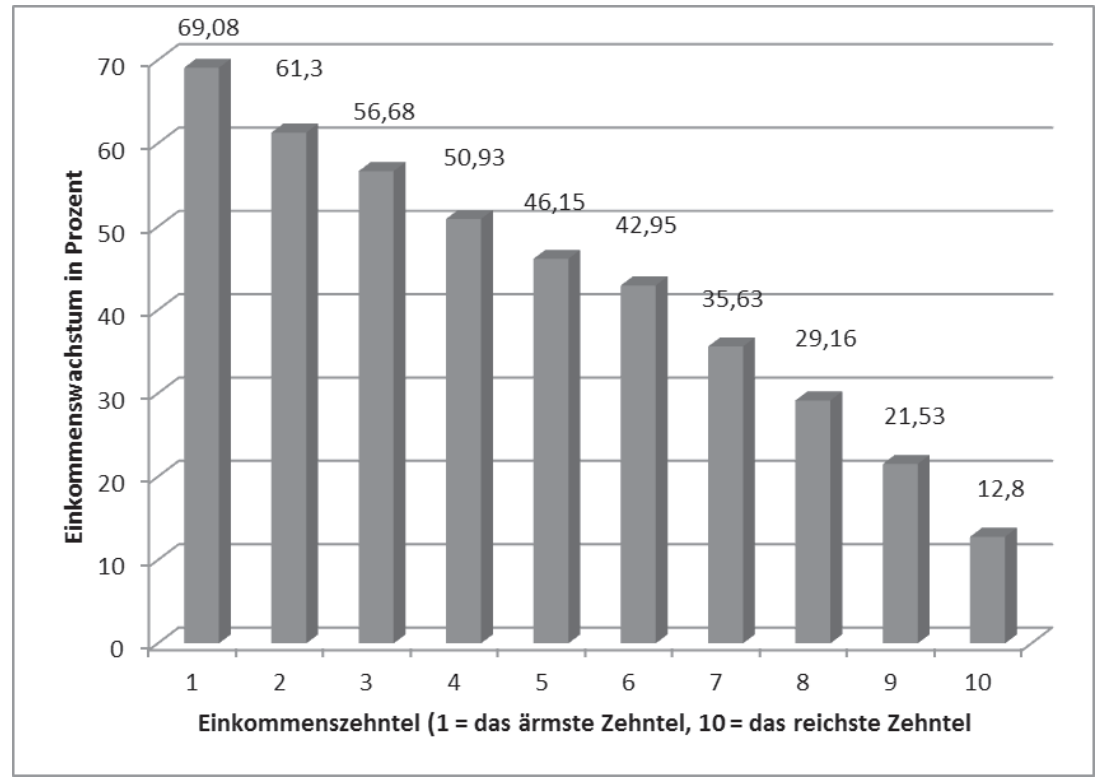

Quelle: Neri 2011: 25.

Das Wachstum zugunsten der Armen war im hochgradig heterogenen Brasilien mehrdimensional. Marcelo Neri (2011: 31ff.) zeigt in seinem Buch A nova classe média. O lado brilhante da base da pirâmide eindrücklich, wie im Zeitraum 2001-2009 die Verringerung der Einkommensunterschiede den benachteiligten Gesellschaftsgruppen systematisch zugutekam. Erstens verbesserte sich das haushaltsbezogene Einkommen pro Kopf $^{1}$ im armen Nordosten Brasiliens um 41,8 Prozent gegenüber 15,8 Prozent im reichen Südwesten. Ein geographisch konkreteres Beispiel: In Maranhão, dem traditionell ärmsten Bundesstaat, stieg das Einkommen um 46,8 Prozent an; in São Paulo, dem reichsten Bundesstaat, lediglich

1 Grundlage dieser Messung ist die Pesquisa Nacional por Amostras de Domicílio (PNAD), die das Gesamteinkommen eines Haushalts bzw. einer Familie unter Berücksichtigung der Anzahl der Mitglieder (also Einkommen pro Kopf) ermittelt. 
um 7,2 Prozent. Zweitens wuchs das Einkommen der Frauen um 38 Prozent, das der Männer nur um 16 Prozent. Drittens nahm das Einkommen derjenigen, die sich selbst als Schwarze bzw. Mischlinge (pretos e pardos) beschreiben, um 43,1 bzw. 48,5 Prozent zu. Das Einkommen der selbstdefinierten Weißen verbesserte sich um 20,1 Prozent. Viertens erfuhren Bürgerinnen und Bürger ohne schulische Bildung eine 46,7-prozentige Erhöhung ihres Einkommens. Diese betrug unter den Menschen mit zumindest unvollständigem oberem Bildungsniveau 17,5 Prozent. Im Falle der Analphabeten entsprach die Verbesserung sogar 53,5 Prozent. Dagegen wuchs das Einkommen derjenigen mit zwölf oder mehr Jahren formaler Bildung 'nur' um 9 Prozent. Zusammengefasst: Die traditionell ausgeschlossenen Bevölkerungssektoren (Schwarze, Analphabeten, Frauen und Personen aus dem Nordosten) sowie Bewohnerinnen und Bewohner der Peripherie und ländlicher Gebiete haben am stärksten von den Einkommenszuwächsen profitiert. Die räumlich bedingte, genderspezifische und ethnische Ungleichheit nahm ab.

\section{Sozialpolitik}

\section{Die Aufwertung der Rolle des Staates}

Derartige Erfolge beim Abbau der sozialen Ungleichheit können nicht ohne die Intervention des Staates erreicht werden. Aus den sozialen Missständen der 1990er Jahre wurde in Lateinamerika die Lehre gezogen, dass wenn eine Gesellschaft durch strukturelle (ökonomische, soziale und politische) Ungerechtigkeit geprägt ist, die (natürliche) Verteilung wachsenden Wohlstands auch ungleichmäßig erfolgt, so dass im besten Falle die Ungleichheit unverändert bleibt bzw. reproduziert wird. Das 'Durchsickern von Reichtum' begünstigt (ungleich) stärker die Privilegierten. Dieser Tendenz kann aber der Staat als aktiver entwicklungspolitischer Akteur entgegenwirken. In unterschiedlichem Ausmaß je nach Land griff dieser in Lateinamerika nun stärker in die Wirtschaft ein, sorgte für (eine induzierte) Umverteilung und nahm damit eine entwicklungspolitische Schlüsselrolle ein. Dieses neue Selbstverständnis wurde gefördert durch zahlreiche Machtwechsel 'gen Links' und einen commodity boom auf dem Weltmarkt. Zum einen setzten die neuen (je nach Fall sozialdemokratischen, sozialistischen, progressiven oder populistischen) Regierungen die soziale Frage hoch auf ihre Agenda. Zum anderen führte die starke (zum 
größten Teil preisbedingte) Expansion der Ausfuhren von Rohstoffen zu Wirtschaftswachstum und der Steigerung der Staatseinnahmen. Daraus ergaben sich der politische Wille und der ökonomische Handlungsspielraum für den Ausbau der staatlichen Sozialausgaben.

\section{Die Bekämpfung der Armut und sozialen Ungleichheit in Brasilien}

Entsprechend dem brasilianischen Verständnis basiert das System von Sozialschutz bzw. sozialer Sicherung (Sistema de Seguridade Social) auf beitragsfreien Leistungen (Assistência Social = Sozialhilfe) und beitragspflichtigen sozialen Versicherungen (Previdência Social $=$ soziale Vorsorge). Die rechtliche Grundlage bildet dabei die brasilianische Verfassung von 1988 (einschließlich der darauffolgenden Reformen bis 2013), in der soziale Rechte (Kapitel II, Artikel 6: Recht auf Bildung, Gesundheit, Ernährung, Arbeit, Wohnung, Erholung, Sicherheit, soziale Vorsorge, Mutter- und Kinderschutz und Sozialhilfe), Arbeitsrechte (Kapitel II, Artikel 7) sowie die Verantwortung des Staates für soziale Leistungen festgeschrieben sind. In diesem Sinne handelt es sich um ein rechtsbasiertes System sozialer Sicherung (Robles/Mirosevic 2013: 7f.). Aus dem Jahr 1993 stammt das Grundgesetz für Sozialhilfe (Lei Orgánica da Assistencia Social, LOAS), das den Schutz der Familien während des ganzen Lebenszyklus und deren Integration in den Arbeitsmarkt und die Gemeinschaft verfolgt. Das Gesetz definiert die Aufgabenteilung zwischen den verschiedenen Ebenen innerhalb der föderalen Staatsorganisation Brasiliens und sieht die Errichtung eines mit Mitgliedern aus der Regierung und der Zivilgesellschaft paritätisch besetzten Nationalen Rates für Sozialhilfe (Conshelo Nacional de Assitência Social) vor. Analoge deliberative Kollektivorgane existieren auf den regionalen und lokalen Ebenen. Ihre Bedeutung kann daran gemessen werden, dass die Nationale Politik für Sozialhilfe des Jahres 2004 (Politica Nacional de Assitência Social, PNAS) ein Resultat deren Arbeit war (Robles/Mirosevic 2013: 19). Im selben Jahr wurde unter der Lula-Regierung als institutioneller Ausdruck des verstärkten politischen Willens zu einer aktiven Sozialpolitik das Ministerium für Soziale Entwicklung und Bekämpfung des Hungers (Ministério do Desenvolvimento Social e Combate à Fome) geschaffen.

Auch wenn die Liberalisierungspolitik der 1990er Jahre in Brasilien nicht die stark negativen sozialen Auswirkungen hatte, die in anderen 
Ländern der Region (etwa in Argentinien, Kolumbien, Peru und Mexiko) beobachtet werden konnten (Robles/Mirosevic 2013: 57), führten erst gezielte Maßnahmen der brasilianischen Regierung eine insbesondere ab 2003 deutlich spürbare stetige Verbesserung der sozialen Lage herbei. Zwar verursachte die globale Finanzkrise 2009 eine Zunahme der Arbeitslosigkeit, der Armut und der sozialen Ungleichheit und somit einen Einbruch im positiven sozioökonomischen Trend. Es ist jedoch davon auszugehen, dass ohne eine ausgeprägte Sozialpolitik die Effekte der Krise gravierender gewesen wären und die darauffolgende Erholung weniger schnell eingesetzt hätte. In diesem Sinne war Brasilien verhältnismäßig gut gewappnet gegen die widrige globale Wetterlage. Bezeichnend ist dabei, dass die Krisensituation (diesmal) nicht eine (prozyklische) Kürzung der Sozialausgaben motiviert hat. Vielmehr wuchsen diese weiterhin, sogar über die Jahre 2008-2010 und wurden durch eine antizyklische Lohnpolitik ergänzt.

Im entwicklungspolitischen Kontext wurden insbesondere jene Programme prominent, die auf konditionierten Finanztransfers basieren. Derartige Maßnahmen wurden zwar bereits unter der Cardoso-Regierung eingeführt. Jedoch verfügte Präsident Lula erst als die Staatseinnahmen als Folge der Einführung des Plano Real erheblich stiegen über die nötigen Ressourcen, um die Sozialausgaben zu erhöhen und die Sozialprogramme auszubauen (Robles/Mirosevic 2013: 7). Voraussetzung hierfür war die Existenz einer signifikanten Steuerquote, das heißt einer (im lateinamerikanischen Vergleich) relativ vollen Staatskasse. Das durch den brasilianischen Staat erhobene Steuervolumen entspricht 32,6 Prozent des BIP, was den höchsten Wert in Lateinamerika (regionaler Durchschnitt: rund 20 Prozent) darstellt und dem OECD-Durschnitt (35 Prozent) sehr nahe kommt (Daten für 2009 aus OECD 2013). Nur ein Staat, der Einnahmen hat, kann sie sozial ausgeben. Zwischen 2002 und 2012 nahmen die Sozialausgaben sowohl als Anteil des BIP als auch der gesamten Staatsausgaben progressiv zu. Zu den Sozialausgaben gehören in Brasilien neben der sozialen Sicherung (Sozialhilfe und soziale Vorsorge) auch Gesundheitsfürsorge, Bildung sowie Wohnungswesen (und sonstige). 
Tabelle 1: Entwicklung von Wachstum, Arbeitslosigkeit und Sozialausgaben, 2002-2012

\begin{tabular}{|c|c|c|c|c|c|}
\hline & $\begin{array}{l}\text { Wachstum } \\
\text { des BIP } \\
\text { in } \%\end{array}$ & $\begin{array}{l}\text { Wachstum } \\
\text { BIP/Kopf } \\
\text { in } \%\end{array}$ & $\begin{array}{l}\text { Arbeits- } \\
\text { losigkeit }\end{array}$ & $\begin{array}{l}\text { Anteil der } \\
\text { Sozialaus- } \\
\text { gaben am } \\
\text { BIP in \% }\end{array}$ & $\begin{array}{l}\text { Anteil der } \\
\text { Sozialaus- } \\
\text { gaben an } \\
\text { den gesam- } \\
\text { ten Staats- } \\
\text { ausgaben } \\
\text { in } \%\end{array}$ \\
\hline 2002 & 2,7 & 1,3 & 11,7 & 21,82 & 68,0 \\
\hline 2003 & 1,1 & $-0,1$ & 12,3 & 22,40 & 71,8 \\
\hline 2004 & 5,7 & 4,5 & 11,5 & 22,30 & 73,3 \\
\hline 2005 & 3,2 & 2,0 & 9,8 & 22,51 & 73,1 \\
\hline 2006 & 4,0 & 2,9 & 10,0 & 23,73 & 73,7 \\
\hline 2007 & 6,1 & 5,1 & 9,3 & 24,41 & 73,2 \\
\hline 2008 & 5,2 & 4,2 & 7,9 & 24,83 & 73,7 \\
\hline 2009 & $-0,3$ & $-1,2$ & 8,1 & 27,06 & 73,5 \\
\hline 2010 & 7,5 & 6,6 & 6,7 & k. A. & k. A. \\
\hline 2011 & 2,7 & 1,9 & 6,0 & k. A. & k. A. \\
\hline $2012^{*}$ & 1,2 & 0,4 & 5,5 & k. A. & k. A. \\
\hline
\end{tabular}

"Vorläufige Daten.

Quelle: CEPALSTAT 2013 
Tabelle 2: Die Entwicklung der Sozialausgaben, 2002-2009

\begin{tabular}{|l|c|c|c|c|c|c|c|c|}
\hline $\begin{array}{l}\text { Anteil am BIP } \\
\text { in } \%\end{array}$ & 2002 & 2003 & 2004 & 2005 & 2006 & 2007 & 2008 & 2009 \\
\hline $\begin{array}{l}\text { Sozialausgaben } \\
\text { insgesamt }\end{array}$ & 21,82 & 22,40 & 22,30 & 22,51 & 23,73 & 24,41 & 24,83 & 27,06 \\
\hline $\begin{array}{l}\text { Soziale } \\
\text { Sicherung }\end{array}$ & 12,34 & 11,59 & 11,93 & 12,28 & 12,89 & 12,79 & 12,81 & 14,07 \\
\hline Bildungswesen & 4,21 & 5,14 & 4,55 & 4,55 & 4,77 & 5,16 & 5,34 & 5,88 \\
\hline $\begin{array}{l}\text { Gesundheits- } \\
\text { fürsorge }\end{array}$ & 3,72 & 4,18 & 4,34 & 4,32 & 4,59 & 4,68 & 4,71 & 5,21 \\
\hline $\begin{array}{l}\text { Wohnungs- } \\
\text { wesen (und } \\
\text { sonstige) }\end{array}$ & 1,56 & 1,49 & 1,48 & 1,37 & 1,57 & 1,77 & 1,97 & 1,90 \\
\hline
\end{tabular}

Quelle: CEPALSTAT 2013

Insgesamt geht in Brasilien die Reduzierung der Armut und der sozialen Ungleichheit nicht nur auf den Ausbau der Sozialhilfe zurück, sondern auch auf die Ausweitung der sozialen Vorsorge sowie auf die Arbeitsmarktpolitik - wie etwa die Steigerung des Mindestlohns (Robles/Mirosevic 2013: 7f.).

\section{Sozialhilfe}

$\mathrm{Zu}$ den beitragsfreien Leistungen gehören die Sozialprogramme konditionierten (an Auflagen gekoppelten) und nicht-konditionierten Finanztransfers. Diese richten sich an Menschen, die in extremer Armut leben (kinderreiche Familien, Schwangere, ältere Menschen ohne Rente etc.). Herkunft und Konzept der Sozialprogramme sind dabei je nach Fall sehr unterschiedlich. Über die Vor- und Nachteile von Finanztransfers als universalistischem Recht vs. konditionierter armutsorientierter Leistung wird international wie in Brasilien noch diskutiert. Durchgesetzt hat sich aber hier eine Armutsfokussierung, die zunächst in der Verantwortung der lokalen Regierungen lag. Das erste Sozialprogramm konditionierten Finanztransfers auf föderaler Ebene wurde 2001 von der Cardoso-Regierung um- 
gesetzt: das Schulstipendium (Bolsa Escola) vom Bildungsministerium. Ihm folgten im selben Jahr das konditionierte Ernährungsstipendium (Bolsa Alimentação) und die nicht-konditionierte Gashilfe (Auxilio Gás). Unter den international bekanntesten sozialpolitischen Strategien Brasiliens, die aus auf Finanztransfers basierten Sozialprogrammen bestehen, befinden sich "Null Hunger" (Fome Zero) und "Brasilien ohne Armut" (Brasil Sem Miséria).

"Null Hunger" ist eine Strategie der brasilianischen Regierung, um das Menschenrecht auf Ernährung zu sichern. Zielgruppe sind Menschen mit eigeschränktem Zugang zu Nahrungsmitteln. Verfolgt wird die Förderung der Nahrungssicherheit, aber auch der sozialen Inklusion und der Bürgerschaft (cidadania) von verwundbaren Bevölkerungsgruppen. Die 2003 unter der Lula-Regierung eingeführte Strategie besteht aus vier thematischen Säulen mit entsprechenden Sozialprogrammen: (1) Zugang zu Nahrungsmitteln; (2) Stärkung der familiären Landwirtschaft; (3) Generierung familiären Einkommens sowie (4) Soziale Rechenschaft und Partizipation (Fome Zero 2013).

"Brasilien ohne Armut" wurde im Jahr 2011 unter der Präsidentschaft von Dilma Rousseff lanciert. Diese sozialpolitische Strategie richtet sich an Haushalte mit weniger als 70 Rais (39 US-Dollar) monatlich und erfasst 16,2 Millionen Menschen bzw. 11,7 Prozent der nationalen Bevölkerung. Sie hat zum Ziel, die extreme Armut bis 2014 gänzlich zu beseitigen. Auch hier sind vier zentrale Aktionsbereiche zu unterscheiden: (1) Einkommensgarantie, in der Regel geleistet durch das Familienstipendium (Bolsa Familia); (2) 'aktive Suche' zur Erfassung von bedürftigen Familien, die im Einheitsregister (Cadastro Único) noch nicht eingetragen sind (also ohne Personalausweis); (3) Eingliederung in den Arbeitsmarkt durch Programme zur Generierung von Einkommen und Beschäftigung in urbanen Regionen (Training, Mikrokredit, Unternehmertum und Arbeitsvermittlung); (4) Zugang zu öffentlichen Dienstleistungen (Brasil Sem Miséria 2013). Im Jahr 2012 wurde im Rahmen der Strategie "Brasilien ohne Armut" das Programm "Liebevolles Brasilien" (Brasil Carinhoso) geschaffen. Es zielt auf die Bekämpfung der Armut unter Kindern ab und beinhaltet wiederum Maßnahmen in den Bereichen Einkommen, Schulbildung und Gesundheit.

Diese sozialpolitischen Strategien sind intersektoral, denn sie integrieren verschiedene Schwerpunktbereiche. Auf diesen Gebieten werden ständig neue Aktionen und Unterprogramme entwickelt. Viele Komponenten (Leistungen/Sozialprogramme) sind an Bedingungen geknüpft. Die Konditiona- 
lität besteht etwa in der Regelmäßigkeit des Schulbesuches von Kindern (85 Prozent Anwesenheit) und Jugendlichen (75 Prozent Anwesenheit), der Ernährungs- und Impfkontrolle von Kindern, des Arztbesuches von Schwangeren etc. Innerhalb der Programme gibt es nach Staatsebene verschiedene Zuständigkeiten. Mittels der Überweisung von Ressourcen und besonderer Prämien an die Gemeinden versucht die Bundesregierung, Anreize für die aktive Beteiligung der lokalen Ebene an der Sozialpolitik zu schaffen.

Als konditioniertes Finanztransferprogramm hat Bolsa Familia (heute als Bestandteil von "Brasilien ohne Armut") den größten Haushalt und die stärkste finanzielle Entwicklung zwischen 2004 und 2011 (von 0,1 auf 0,4 Prozent des BIP) erfahren. Es weist zudem den umfassendsten Deckungsgrad auf: Im Jahr 2011 waren 27,4 Prozent der brasilianischen Bevölkerung (mehr als 54 Millionen) Nutznießer des Programms (Robles/ Mirosevic 2013: 25). Dank der Fokussierung auf die unteren Zehntel der Einkommenspyramide haben das Familienstipendium und ähnliche Programme nicht nur zur Armutsbekämpfung, sondern auch zum Abbau der sozialen Ungleichheit beigetragen.

\section{Soziale Vorsorge}

Zentrale Säule der sozialen Vorsorge ist in Brasilien die Altersvorsorge. Diese besteht heute aus zwei Untersystemen: Das Sonderregime der Pensionsvorsorge (Regime Própio de Previdência Social, RPPS), mit vergleichbar höheren Renten für Beamte und Militärs (keine Arbeiter oder Angestellte des privaten Sektors sind im System) und das Allgemeine Regime der Pensionsvorsorge (Regime Geral de Previdência Social, RGPS) für alle übrigen Arbeitnehmer und Arbeitnehmerinnen. Das Pensionsalter variiert je nach System, Gebiet (urban/ländlich) und Geschlecht zwischen 55 und 70 Jahren. Die erforderliche Mindestzeit für Beitragszahlungen beträgt zwischen 30 und 35 Jahre. Beide Regime beinhalten eine solidarische Komponente der intergenerationalen und interregionalen Umverteilung und sind gemischt finanziert: Der Staat, die Arbeitgeber und die Arbeitnehmer leisten Beiträge. Deren Verwaltung obliegt einer staatlichen Anstalt (Instituto Nacional do Seguro Social, INSS) (Robles/Mirosevic 2013: 31). Zusätzlich zu diesen zwei Rentensystemen gibt es ein freiwilliges Regime nach dem pay-as-you-go Prinzip. Es wird privat verwaltet durch profitorientierte und nicht-profitorientierte Institutionen. 
Die Verfassung von 1988 definierte den Mindestlohn als Grundlage jeglicher Leistung der sozialen Sicherung. Ende der 2000er Jahre wurde eine jährliche Mindestlohnanpassung auf der doppelten Grundlage des Inflationsausgleichs und des Wirtschaftswachstums festgelegt. Folglich dürfen Renten - der erfolgten Beitragszahlungen ungeachtet - nicht unter dieses Niveau fallen. Im Dezember 2010 entsprachen 99,3 Prozent der Gesamtheit der in ländlichen Gebieten gezahlten Renten dem Mindestlohn; sie erreichten 8,2 Millionen direkte Leistungsberechtigte. In den Städten waren 46 Prozent der insgesamt bezahlten Renten auf dem Niveau des Mindestlohns; sie erfassten 7,4 Millionen Empfängerinnen und Empfänger (Robles/Mirosevic 2013: 31).

Diese Werte offenbaren zweierlei: Zum einen bezieht der größte Teil der Leistungsberechtigten die niedrigste Rente. Aus diesem Grund ist zum anderen die Festlegung des Mindestlohns als 'Rentenboden' besonders sozial relevant. Insgesamt wirkt im System ein Umverteilungsmechanismus, der zu Einkommenssteigerungen bei den ärmsten Bevölkerungsgruppen führt. Seit 2002 steigt der Anteil der ökonomisch aktiven Menschen stetig an, die durch das System erfasst werden. Im Zeitraum 2002-2009 wuchs er von 53,8 auf 59,3 Prozent. Hierfür sind in erster Linie das Wirtschaftswachstum und die zunehmende Formalisierung des Arbeitsmarktes verantwortlich. Dennoch besteht ein Missverhältnis zwischen Einzahlungen und Leistungen, das der Staat im Sinne der Sicherung einer Mindestrente und der Angleichung zwischen urbanen und ländlichen Gebieten aus eigener Kraft kompensieren muss. Im Jahr 2010 entsprachen die Beiträge zum Allgemeinen Regime der Pensionsvorsorge 5,8 Prozent des BIP, die Ausgaben für die Leistungen aber 7 Prozent. 1,2 Prozent des BIP waren folglich notwendig, um das Defizit abzudecken (Robles/Mirosevic 2013: 34).

Die Systeme der sozialen Sicherung bilden den zweitwichtigsten Erklärungsfaktor für den Abbau der Ungleichheit in Brasilien. Hierzu gehört auch die Beihilfe der Fortgesetzten Leistung (Beneficio de Prestação Continua$d a, \mathrm{BPC}$ ), ein Mindesteinkommen für ältere Menschen ab 65 Jahren (Robles/Mirosevic 2013: 7f.). Der Beitrag der sozialen Sicherung zur Ungleichheitsreduzierung im Zeitraum 2001-2011 wird auf 19 Prozent geschätzt (IPEA 2012). Einen bedeutenden Effekt in diesem Zusammenhang hatte zudem die Entwicklung der Beschäftigung. 


\section{Arbeit}

Da der Arbeitsmarkt in Lateinamerika für 70 Prozent des gesamten Einkommens verantwortlich ist, haben Veränderungen in diesem Bereich starke Effekte auf die Einkommensverteilung (Gasparini/Cruces 2013: 55). Relevante Akteure der brasilianischen "Dekade der Inklusion" (IPEA 2012) waren nicht nur die Regierungen der Arbeiterpartei. Dabei spielten auch die im lateinamerikanischen Vergleich mächtigen brasilianischen Gewerkschaften eine Rolle. Sie verstehen sich als Teil der politischen Bewegung, die Lula und Dilma an die Regierung brachte und üben auch Druck auf das Regierungshandeln aus (Zilla 2012: 47). Die brasilianische Wirtschaftspolitik ist seit 2003 durch eine Nachfrageorientierung gekennzeichnet. Sie basiert auf der Erhöhung des Mindestlohns, die sowohl Rentenanpassungen als auch Einkommenszuwächse zur Folge hat. Zwischen 1995 und 2005 stieg der Mindestlohn um 3,8 Prozent jährlich. Im Jahr 2007 wurde dieser durch eine politische Maßnahme an die Veränderung des BIP und die Inflation gekoppelt; im Jahr 2011 wurde diese Bindung per Gesetz festgeschrieben. Anfang 2009 wurde durch eine antizyklische Entscheidung der Mindestlohn nominell um zwölf bzw. real um sechs Prozent erhöht. Im Januar 2010 betrug der Mindestlohn 510 Real $(=274$ US-Dollar). Insgesamt erfuhr der Mindestlohn zwischen 2005 und 2011 eine jährliche reale Zunahme von drei Prozent. Dieser Art Politik wird ein starker Umverteilungseffekt zugeschrieben, da der Mindestlohn von den großen unterprivilegierten Bevölkerungssektoren bezogen wird. Laut Studien ist er im Zeitraum 1995-2005 für 73 Prozent der Verbesserung in der Einkommensverteilung verantwortlich (Robles/Mirosevic 2013: 14).

Zur Verringerung der Einkommensungleichheit hat auch die Reduzierung des Einkommensunterschieds zwischen qualifizierter und nicht qualifizierter Arbeit (sogenannte Gehaltsprämie) beigetragen. Die Gehaltsprämie (also der Vorteil qualifizierter Arbeit) erhöhte sich in den 1990er Jahren durchschnittlich um 1,8 Prozent pro Jahr, während sie sich im vergangenen Jahrzehnt um 2,8 Prozent reduzierte. Veränderungen der Gehaltsprämie werden durch das Angebot und die Nachfrage von Arbeit sowie durch Arbeitsmarktpolitik induziert.

Die Beschäftigungspolitik hat sich in Brasilien traditionell auf vier Bereiche konzentriert: Arbeitslosenversicherung, Stellenvermittlung, Berufsbildung und Mikrokredit. Hierbei spielen Maßnahmen des Arbeitsministeriums (Ministério do Trabalho e Emprego, MTE) eine zentrale Rolle, 
deren Zielgruppe Arbeitslose sind. Besonders benachteiligte Gruppen mit erschwertem Zugang zum Arbeitsmarkt werden eher durch Sozialprogramme (Sozialhilfe) unterstützt, etwa im Rahmen der Strategien "Null Hunger" und "Brasilien ohne Armut" (Robles/Mirosevic 2013: 49). Diese beinhalten zahlreiche arbeitsmarktbezogene Pläne, Programme und Aktionen, die in der letzten Dekade vermehrt eingeführt wurden.

\section{Defizite und Herausforderungen}

Nach Studien des brasilianischen Instituts für Angewandte Wirtschaftsforschung ist im Zeitraum 2001-2011 das Einkommen jener Menschen, die zum ärmsten Zehntel gehören, 550 Prozent mehr gewachsen als das des reichsten Zehntels. Zwischen 2001 und 2008 wird die Gini-Reduzierung zu 58 Prozent durch Effekte der Arbeit, zu 19 Prozent durch Leistungen der sozialen Vorsorge und zu 13 Prozent durch Bolsa Família erklärt. Jeder Prozentpunkt der Reduzierung von Gini, der durch die soziale Vorsorge herbeigeführt wurde, kostete 532 Prozent mehr als im Falle des Sozialprogramms. Eine größere Ungleichheitsverminderung wäre also erreicht worden, hätte man sich mit denselben Ressourcen mit einer stärkeren Armutsorientierung auf Bolsa Família konzentriert (IPEA 2012: 38f.). Heute stellt das Familienstipendium 0,5 Prozent des BIP dar.

Diese Schlussfolgerung legt den Fokus auf Einkommensunterschiede, blendet jedoch politische und soziale Aspekte aus. Wie wird Bürgerschaft (cidadania) in dem einen und anderen Fall verwirklicht und erlebt? Was ist das Selbstverständnis einer Bürgerin bzw. eines Bürgers als leistungsberechtigter Person der sozialen Vorsorge einerseits und der Sozialhilfe andererseits? Sind da Unterschiede auszumachen? Dies sind Fragen, die über die Ungleichheit hinaus die soziale Gerechtigkeit sowie die Beziehungen der Bürgerinnen und Bürger mit dem Staat und deren Status in der Gesellschaft betreffen. Es fehlt an Analysen, welche diese soziopolitischen und sozialpsychologischen Dimensionen der soziökonomischen Errungenschaften Brasiliens zum Gegenstand machen.

Die Einkommenssteigerungen haben die Kaufkraft der Armen erhöht und durch finanzielle Anreize den Zugang zu öffentlichen Dienstleistungen erweitert (= Quantität). Das staatliche Bildungs- und Gesundheitswesen weist aber noch gravierende Defizite auf, welche die Chancengleichheit beeinträchtigen (= Qualität). Bezüglich der Ergebnisse der Grundschul- 
bildung befindet sich Brasilien auf dem 127. Platz im PISA-Bericht des Jahres 2010 und liegt somit deutlich hinter China (Platz 35) (Trebat 2013: 131). Auch die Qualitätssteigerung des Gesundheitswesens und die Errichtung eines Netzes öffentlicher Gesundheitsstationen blieben bisher weit hinter den Wahlversprechen von Präsidentin Dilma Rousseff zurück.

Für die Verstetigung der Ungleichheit sorgt in Brasilien zudem das Steuersystem. Brasilien ist zwar der lateinamerikanische Staat mit den höchsten Steuereinnahmen. Das Geld erhält der Staat aber vor allem über die Mehrwertsteuer, welche im Unterschied zu den direkten Abgaben einen regressiven Effekt hat. Sie belastet die niedrig Verdienenden stärker. Die Einkommensungleichheit wird entsprechend durch das Steuersystem reproduziert, eine fiskalische Umverteilungswirkung ist kaum vorhanden (OECD 2012). Im Grunde sind es die direkten Finanztransfers des Staates, welche diese steuerpolitische Dysfunktionalität zu kompensieren vermögen (Zilla 2012: 48f.).

Die nachfrageorientierte Wirtschafts- und Sozialpolitik fördert den Konsum. Die brasilianische Gesellschaft ist insgesamt stark auf Konsum ausgerichtet - während die Sparquote und die Investitionsrate, die nachhaltige Entwicklung gewährleisten sollten, nach wie vor nicht hoch genug sind. Vor allem die Investitionen in Wissenschaft, Technologie und Innovation sind weiterhin sehr niedrig, sie entsprechen 1,1 Prozent des BIP, und im Unterschied zu den OECD-Ländern, ist hierfür in erster Linie der Staat und nicht die Privatwirtschaft verantwortlich (OECD 2012). Die Unternehmen engagieren sich ungenügend in der Förderung der angewandten Wissenschaften. Das alles trägt zur niedrigen Produktivität und Wettbewerbsfähigkeit Brasiliens bei. Dies stellt kein großes Problem dar, solange die Volkswirtschaft relativ geschlossen (der Außenhandel entspricht lediglich 25 Prozent des BIP) bzw. protektionistisch bleibt und der Binnenmarkt Wachstumspotential besitzt. Die Schattenseite dieser Entwicklung sind aber erhöhte Preise und/oder Produkte niedriger Qualität für die Verbraucherinnen und Verbraucher.

In diesem Sinne ist in Brasilien der Binnenmarkt für das stete Wirtschaftswachstum seit 2003 verantwortlich - und nicht etwa der Außenhandel. Im Unterschied zu anderen Schwellenländern wirkte auch nicht eine hohe Investitionsrate als Konjunkturmotor, sondern eine starke Nachfrage nach lang- und kurzlebigen Konsumgütern. Durch erhebliche Einkommenssteigerungen wurden sie zugänglich insbesondere für Bevölkerungsgruppen, die einst in Armut gelebt hatten und nun in die 
Mittelschicht aufstiegen (Zilla 2012: 50f.). Im Kontrast zu vielen Schwellenländern, in denen die soziale Schere auseinander ging, hat Brasilien es allerdings geschafft, die soziale Ungleichheit stark zu verringern.

Eine nachhaltige gerechte Wohlfahrtsentwicklung bedarf aber eines komplexeren, umfassenden Ansatzes, der mehr als die Steigerung der Kaufkraft in den Blick nimmt. Die erfolgreiche brasilianische Armutsund Ungleichheitsbekämpfungsstrategie ist stark auf die Bereitstellung von Finanzmitteln angewiesen. Nach der verlorenen Dekade der 1980er Jahre waren die 1990er Jahre durch eine Politik der Wirtschaftsstabilisierung gekennzeichnet. Die 2000er Jahre waren dank der Reduzierung der Armut und der sozialen Ungleichheit ein für die Inklusion gewonnenes Jahrzehnt. Mögen in der nahen Zukunft die nötigen strukturellen Reformen erfolgen, die längerfristig ein Ministerium für soziale Entwicklung und Bekämpfung des Hungers überflüssig werden lassen.

\section{Literatur}

Brasil SEM Miséria (2013): <http://www.brasilsemmiseria.gov.br/> (30.05.2013).

Cepalstat (2013): Comisión Económica para América Latina y el Caribe, División de Estadísticas. Unidad de Estadísticas Sociales.

Fome Zero (2013): <http://www.fomezero.gov.br/> (30.05.2013).

Gasparini, Leonardo/Cruces, Guillermo (2013): "Poverty and Inequality in Latin America: A Story of Two Decades". In: Journal of International Affairs, Spring/Summer, 66, 2, 51-63.

IPEA (2012): A Década Inclusiva (2001-2011): Desigualdade, Pobreza e Políticas de Renda, Comunicados de IPEA, Nr. 155, Brasília: Instituto de Pesquisa Econômica Aplicada.

Lustig, Nora/López-Calva, Luis F./Ortiz-Juárez, Eduardo (2012): Declining Inequality in America in the 2000s: The Cases of Argentina, Brazil, and Mexico. Working Paper 307. Washington: Center for Global Development.

Neri, Marcelo (2011): A nova classe média. O lado brilhante da base da pirâmide. São Paulo: Saraiva.

OECD (2012): Latin American Economic Outlook 2012. Organisation for Economic Co-operation and Development.

- (2013): Country statistical profile: Brazil 2013. Organisation for Economic Co-operation and Development. <http://www.oecd-ilibrary.org/economics/country-statisticalprofile-brazil-2013_csp-bra-table-2013-1-en> (30.05.2013).

Robles, Claudia/Mirosevic, Vlado (2013): Social Protection Systems in Latin America and the Caribbean: Brazil. Santiago de Chile: Economic Commission for Latin America and the Caribbean (ECLAC). 
Trebat, Thomas (2013): "New Directions for a More Prosperous Brazil". In: Journal of International Affairs, Spring/Summer, 66, 2, 127-142.

WeLtBAnK (2011): <http://data.worldbank.org/country/brazil> (30.05.2013)

Zilla, Claudia (2012): “Sozioökonomische Inklusion. Neue linke Sozialpolitik". In: WeltTrends, Zeitschrift für Internationale Politik, 85, Juli/August, 20 Jahrgang, 44-51.

Zilla, Claudia/Harig, Christoph (2012): Brasilien als "Emerging Donor". Politische Distanz und operative Nähe zu den traditionellen Gebern. Berlin: SWP- Studien 2012/S07. 